\title{
Demographic, Endoscopic and Histopathologic Features Among Stool $H$. pylori Positive and Stool $H$. pylori Negative Patients With Dyspepsia
}

\author{
Mohamed Hassan Emara a, b, d, Rasha Ibrahim Salama ${ }^{a}$, \\ Amira Amin Salem ${ }^{c}$
}

\begin{abstract}
Background: Dyspepsia is a common presentation for many patients in gastroenterology clinics. Helicobacter pylori (H. pylori) infection is endemic in many countries around the world and its relation to dyspepsia has long been questioned. This study aimed at comparing demographic, endoscopic and histologic features among dyspeptic patients with and without stool H. pylori antigen positivity.
\end{abstract}

Methods: One hundred and fifty-one consecutive patients with dyspepsia were divided into group I $(\mathrm{n}=80)$ and group II $(\mathrm{n}=71)$ according to positive or negative $H$. pylori stool antigen testing, respectively. All patients were subjected to history taking, clinical examination, laboratory investigations, abdominal ultrasonography, $H$. pylori stool antigen detection, and upper gastrointestinal tract endoscopy with four gastric mucosal biopsies for histopathologic examination.

Results: Stool H. pylori antigen negative group included more smokers and consumed much more fast and spicy food than the positive group. The most common endoscopic findings of both groups were gastritis, reflux esophagitis, gastric erosions, peptic ulcer, mucosal nodularity and hiatus hernia and were encountered in $100 \%$, $74.2 \%, 23.2 \%, 15.2 \%, 13.9 \%$, and $13.2 \%$ respectively with nonsignificant difference between both groups except for gastric erosions that were significantly higher in stool $H$. pylori negative group $(\mathrm{P}<0.001)$. Histologic evidence of gastritis was reported in $100 \%$ and $92.9 \%$ of patients in group I and group II, respectively. Chronic active gastritis with neutrophil infiltration was significantly higher in group I $(\mathrm{P}<0.001)$. H. pylori bacilli were histologically detected

Manuscript submitted July 13, 2017, accepted August 31, 2017

${ }^{a}$ Tropical Medicine Department, Faculty of Medicine, Zagazig University, Zagazig, Egypt

${ }^{b}$ Hepatology, Gastroenterology and Infectious Diseases Department, Faculty of Medicine, Kafrelsheikh University, Kafrelsheikh, Egypt

'Pathology Department, Faculty of Medicine, Zagazig University, Zagazig, Egypt

${ }^{\mathrm{d} C o r r e s p o n d i n g ~ A u t h o r: ~ M o h a m e d ~ H . ~ E m a r a, ~ T r o p i c a l ~ M e d i c i n e ~ D e p a r t m e n t, ~}$ Faculty of Medicine, Zagazig University, Zagazig 44519, Egypt; Hepatology, Gastroenterology and Infectious Diseases Department, Faculty of Medicine, Kafrelsheikh University, Kafrelsheikh, Egypt.

Email: emara_20007@yahoo.com

doi: https://doi.org/10.14740/gr886w in $83.7 \%$ and $47.9 \%$ of patients in group I and group II, respectively $(\mathrm{P}<0.001)$.

Conclusion: All patients with dyspepsia in this study had endoscopic evidence of gastritis. Most of these patients had histologic evidence of gastric mucosal inflammation. Consequently, it may be advisable to perform endoscopy on these patients and obtain gastric mucosal biopsies.

Keywords: Helicobacter pylori; Dyspepsia; Endoscopy; Gastritis

\section{Introduction}

Dyspepsia is a clinical syndrome including manifestations related to the upper gastrointestinal tract (GIT), which may include acute, chronic, or recurrent pain or discomfort. Other associated symptoms include fullness, early satiety, and bloating, burning, belching, nausea, retching, and vomiting. It is generally categorized into functional and organic dyspepsia. In the functional dyspepsia, no obvious cause is detected. Functional dyspepsia is classified after Rome III criteria into two major subtypes, the post-prandial distress syndrome and epigastric pain syndrome with criteria fulfilled in the last 3 months and symptoms onset at least 6 months prior to diagnosis, again in the absence of structural disease $[1,2]$. The most common causes of organic dyspepsia include peptic ulcer disease, gastritis, hepatobiliary disorders, gastroesophageal reflux disease (GERD) and malignancy. The burden of dyspepsia on the daily medical service is huge, because its prevalence in the general population varies from $25 \%$ to $40 \%$ and accounts for $5 \%$ of general clinics visits [1].

Helicobacter pylori (H. pylori) infection is a widespread disease and is endemic in many countries around the world especially in the developing countries [3] with a wide range of morbidity, ranging from asymptomatic colonization of the gastric mucosa, through acute gastritis, chronic gastritis, atrophic gastritis that may be complicated by intestinal metaplasia, dysplasia and gastric cancer and that is why it needs recognition and appropriate therapy [4].

This study was conducted to reveal different demographic, endoscopic and histologic features among dyspeptic patients with and without stool $H$. pylori antigen positivity. 


\section{Patients and Methods}

\section{Study design}

This was a cross-sectional observational and interventional study.

\section{Patients}

All adult patients with dyspepsia who presented to Outpatient Gastroenterology Clinics of the Tropical Medicine Department, Faculty of Medicine, Zagazig University Hospitals, Egypt in the period from March 2016 to March 2017 were offered to participate in the study. Patients who agreed gave a written informed consent for participating in the study. One hundred fifty-one consecutive patients were enrolled and were assigned into two groups: group I - stool H. pylori antigen positive group; group II - the stool $H$. pylori antigen negative group.

\section{Inclusion criteria}

Patients with these criteria were included: 1) age range from 18 to 60 years; 2 ) both male and female; 3 ) good mentality to understand aim, benefits and steps of the study; 4) assumed availability during the study period; and 5) agreement upon examination by upper endoscopy and biopsy.

\section{Exclusion criteria}

Patients with these conditions were excluded from the study: 1) chronic diseases, e.g. diabetes, renal failure, and cirrhosis; 2) malignancy; 3) gallbladder disorders; 4) prior peptic ulcer diseases; 5) prior upper GIT surgery; and 6) patients who used proton pump inhibitors (PPIs), antibiotics or probiotics within 4 weeks before being enrolled in the study

\section{Definitions}

H. pylori infection is defined in this study when the patient was positive or negative for $H$. pylori antigen in stool provided that he did not receive any PPIs or antibiotics in the last 4 weeks prior to the examination.

Dyspepsia is a term used for acute, chronic, or recurrent pain or discomfort centered in the upper abdomen. It may be associated with upper abdominal fullness, early satiety, and bloating, burning, belching, nausea, retching, and vomiting [1].

Endoscopic examination was performed to all patients after explanation about the procedure, possible complications, benefits and biopsy. All patients were examined under light sedation in the left lateral position. A CD video was obtained for every patient for documentation and possible re-evaluation if needed. Complete report about the esophageus, stomach and duodenum till the second part was obtained. Endoscopic gastritis was defined when the mucosa of the stomach was apparently inflamed with erythema and nodularity [5]. Four samples were taken, two from the antrum and two from the body of the stomach for histologic examination [5].

Histopathologic analysis was performed for all biopsies with hematoxylin and eosin and Giemsa stains. All biopsy specimens were evaluated for the presence of $H$. pylori bacilli, mononuclear cells (lymphocytes, plasma cells and histiocytes) as markers of inflammation and polymorphonuclear leucocytes (neutrophils) as markers of activity. Inflammation and activity were graded using a semi-quantitative score ranging from 0 (none), 1 (minimal), 2 (mild), 3 (moderate) to 4 (severe) [5].

\section{Patient assessment}

All patients were subjected to: 1) full history taking; 2) full clinical examination; 3) abdominal ultrasound examination; and 4) stool H. pylori antigen (On Site H. pylori Ag Rapid Test, CTK Biotech, USA). It is a lateral flow chromatographic immunoassay for qualitative assessment of $H$. pylori antigens depending on the use of monoclonal antibodies against $H$. $p y$ lori conjugated with colloid gold. Positive cases were marked with two bands of color changes (one test band and one control band). 5) upper GIT endoscopy with full comment on endoscopic lesions and biopsy; and 6) histopathologic examination of the gastric biopsies (hematoxylin and eosin and Giemsa stains) for determining the presence of $H$. pylori bacilli, presence and density of inflammatory cells including lymphocytes, plasma cells and neutrophils.

\section{Statistical analysis}

Data were analyzed using SPSS version 16. Data were expressed as number and percentage for qualitative variables and mean \pm standard deviation for quantitative ones. P value of $<$ 0.05 indicates significant results. Comparison between the two groups was done using $t$-test and Chi-square test.

\section{Ethical considerations}

The study protocol was approved by the Institutional Review Board of the Faculty of Medicine, Zagazig University, Egypt. All patients gave a written informed consent for participating in the study and for performing all relevant interventions. The study protocol was adherent to practice guidelines and Declaration of Helsinki.

\section{Results}

\section{Study patients}

One hundred fifty-one patients were consecutively enrolled. Group I comprised 80 patients with confirmed $H$. pylori infec- 
Table 1. Baseline Characteristics of Both Groups

\begin{tabular}{|c|c|c|c|c|c|}
\hline & \multicolumn{2}{|c|}{ Group I $(N=80)$} & \multicolumn{2}{|c|}{ Group II $(N=71)$} & \multirow{2}{*}{ P value } \\
\hline & No. & $\%$ & No. & $\%$ & \\
\hline Age $($ mean $\pm \mathrm{SD})$, range & \multicolumn{2}{|c|}{$37.1 \pm 9.5(20-56)$} & \multicolumn{2}{|c|}{$37 \pm 9.4(20-64)$} & 0.972 \\
\hline Sex & & & & & 0.869 \\
\hline Male & 44 & 55.0 & 40 & 56.3 & \\
\hline Female & 36 & 45.0 & 31 & 43.7 & \\
\hline Smoking & 22 & 27.5 & 37 & 52.1 & 0.002 \\
\hline Socioeconomic status & & & & & 0.623 \\
\hline Low & 46 & 57.5 & 38 & 53.5 & \\
\hline High & 34 & 42.5 & 33 & 46.5 & \\
\hline Education & & & & & 0.831 \\
\hline Low & 46 & 57.5 & 38 & 53.5 & \\
\hline Secondary & 30 & 37.5 & 30 & 42.3 & \\
\hline High & 4 & 5.0 & 3 & 4.2 & \\
\hline Diet & & & & & 0.027 \\
\hline Spicy & 34 & 42.5 & 43 & 60.6 & \\
\hline Regular & 46 & 57.5 & 28 & 39.4 & \\
\hline
\end{tabular}

tion using the readily available stool $H$. pylori antigen, while groups II comprised 71 patients who were negative for $H$. $p y$ lori stool antigen. The baseline characteristics of both groups are shown in Table 1. There had been no significant differences between both groups for pre-enrollment criteria except that group II included more smokers $(\mathrm{P}=0.002)$ and had higher frequency of fast and spicy food consumption $(\mathrm{P}=0.027)$ than group I.

\section{Endoscopic features}

The most common endoscopic findings (Table 2) of both groups were gastritis, reflux esophagitis, gastric erosions, peptic ulcer, mucosal nodularity and hiatus hernia and were encountered in $100 \%, 74.2 \%, 23.2 \%, 15.2 \%, 13.9 \%$, and $13.2 \%$, respectively with non-significant difference between both groups except for gastric erosions (superficial mucosal injury) that were significantly higher in stool $H$. pylori negative group $(\mathrm{P}<0.001)$.

Gastritis at the time of endoscopy was the most common endoscopic finding in this study and was detected in $100 \%$ of patients and is either pan-gastritis $(\mathrm{n}=133,88 \%)$, atrophic gastritis $(n=9,6 \%)$ or antral predominant gastritis $(n=9,6 \%)$. Reflux esophagitis was the second common endoscopic finding in this study and was reported in $112(78.8 \%)$ of all patients and most of the patients were in grade A. No malignancy was detected in either group.

\section{Histopathology features}

All patients of group I ( $100 \%)$ had histologic evidence of gastritis, while most of the stool $H$. pylori negative patients $(92.9 \%)$ had this histologic evidence of gastritis. In the stool $H$. pylori positive group, chronic active gastritis was the most common histologic finding $(96.3 \%)$ characterized by the neutrophils infiltration within the gastric mucosa. On the other hand, patients with negative stool $H$. pylori testing showed picture of chronic inactive gastritis (infiltration of the gastric mucosa by plasma cells and lymphocytes). The most surprising finding here is that not all stool $H$. pylori positive patients $(\mathrm{n}=80)$ showed detectable $H$. pylori bacilli (67 patients, $83.8 \%$ ) in their histology examination and this may point to patchy distribution of the organism and the need for more endoscopic biopsies. The other surprising finding is that despite testing negative for stool $H$. pylori antigen $(\mathrm{n}=71)$ still 34 patients $(47.9 \%)$ of the group II had the bacilli seen in their histology examination. Advanced stages of gastric mucosal pathology including intestinal metaplasia and dysplasia were detected only in a minority of patients $(0.7 \%$ and $5.3 \%$, respectively). Erosions of the surface mucosal epithelium are a common feature in both groups. It is significantly higher in group II (35.2\%) than group I $(12.5 \%)(\mathrm{P}<0.001)$. It is obvious that stool H. pylori positive group had severe histologic parameters than the negative group and this is shown by significant difference in most of the histologic features (Table 3 ).

\section{Discussion}

The worldwide prevalence of dyspepsia is high [6] and that is why it is part of daily clinical practice to see many patients with dyspepsia. The decision to proceed to endoscopy in these patients depends on presence or absence of alarm manifestations proposed by the American Gastroenterological Association (AGA) [7]. These criteria include the unintended weight 
Table 2. Endoscopic Features of Both Groups

\begin{tabular}{|c|c|c|c|c|c|}
\hline & \multicolumn{2}{|c|}{ Group I $(\mathbf{N}=\mathbf{8 0})$} & \multicolumn{2}{|c|}{ Group II $(\mathrm{N}=71)$} & \multirow{2}{*}{ P value } \\
\hline & No. & $\%$ & No. & $\%$ & \\
\hline \multicolumn{6}{|l|}{ Reflux esophagitis } \\
\hline A & 46 & 57.5 & 47 & 66.2 & 0.273 \\
\hline B & 8 & 10.0 & 6 & 8.5 & 0.743 \\
\hline $\mathrm{C}$ & 0 & 0.0 & 1 & 1.4 & 0.287 \\
\hline $\mathrm{D}$ & 4 & 5.0 & 0 & 0.0 & 0.056 \\
\hline Hiatus hernia & 10 & 12.5 & 8 & 11.3 & 0.816 \\
\hline \multicolumn{6}{|l|}{ Gastritis } \\
\hline Pan-gastritis & 70 & 87.5 & 63 & 88.7 & 0.816 \\
\hline Atrophic & 4 & 5.0 & 5 & 7.0 & 0.597 \\
\hline Antral predominant & 6 & 7.5 & 3 & 4.2 & 0.396 \\
\hline Erosions & 10 & 12.5 & 25 & 35.2 & $<0.001$ \\
\hline Peptic ulcer & 12 & 15.0 & 11 & 15.5 & 0.933 \\
\hline Mucosal nodularity & 12 & 15.0 & 9 & 12.7 & 0.68 \\
\hline
\end{tabular}

loss, progressive dysphagia, persistent vomiting, and evidence of gastrointestinal bleeding with family history of cancer. The AGA recommends endoscopy to be done first in patients with alarm signs and in patients $>55$ years old. It is generally accepted to begin empiric PPIs therapy in patients lacking these alarm manifestations and also to test and treat for $H$. pylori if the prevalence is high. In Egypt, like most of the developing countries, the prevalence of $H$. pylori infection is high, and the rate is higher in patients with dyspepsia, in patients with histologically confirmed gastritis and in patients of older age groups $[8,9]$ and that is why testing for $H$. pylori is routinely performed for dyspeptic patients.

Due to limited availability and expense of urea breath test (UBT) especially in small towns and rural areas, we usually in the daily practice rely on $H$. pylori stool antigen detection for diagnosis of $H$. pylori infection and that is why this test is used in this study for diagnosis of $H$. pylori infection, to reflect real life scenario that we usually do.

It is evident from the results of the current study that all dyspeptic patients have endoscopic findings ranging from simple gastritis to GERD and peptic ulcer. In fact, according to these findings, it may be of value to proceed to upper endos- copy and probably also biopsy in dyspeptic patients even if the alarm manifestations were lacking. Similar findings were reported by other authors in our community $[10,11]$ and also from other parts of the world where H. pylori is also of high prevalence $[12,13]$ with increasing age [11].

The most common endoscopic findings in both groups were gastritis, GERD, gastric erosions, peptic ulcer, mucosal nodularity and hiatus hernia, respectively; similar results were also reported from East African Coast in Tanzania [12]. However, a large retrospective study from Egypt including 1,400 patients found that peptic ulcer, esophagitis, and erosive gastroduodenitis were the most common endoscopic findings. Of note, $32 \%$ of patients in this study had history of smoking, $32 \%$ were taking aspirin or non-steroidal anti-inflammatory drugs and $2 \%$ were consuming alcohol and this explains the differences with our study [11].

All patients of this study had morphologic evidence of gastritis and there was an agreement between endoscopic findings and histological features. All patients in group I (100\%) had histological features of chronic gastritis and 93\% of patients in group II had also histological features of gastritis. The point of agreement between endoscopic and histologic features

Table 3. Histopathology Features of Both Groups

\begin{tabular}{|c|c|c|c|c|c|}
\hline & \multicolumn{2}{|c|}{ Group I $(\mathrm{N}=\mathbf{8 0})$} & \multicolumn{2}{|c|}{ Group II $(N=71)$} & \multirow{2}{*}{ P value } \\
\hline & No. & $\%$ & No. & $\%$ & \\
\hline Chronic active gastritis & 77 & 96.3 & 19 & 26.8 & $<0.001$ \\
\hline Chronic inactive gastritis & 3 & 3.8 & 47 & 66.2 & $<0.001$ \\
\hline H. pylori bacilli present & 67 & 83.8 & 34 & 47.9 & $<0.001$ \\
\hline Dysplasia & 8 & 10.0 & 0 & 0.0 & 0.006 \\
\hline Intestinal metaplasia & 0 & 0.0 & 1 & 1.4 & 0.287 \\
\hline Erosive gastritis & 46 & 57.5 & 27 & 38.0 & 0.017 \\
\hline Glandular atrophy & 3 & 3.8 & 0 & 0.0 & 0.099 \\
\hline
\end{tabular}


was questioned. One study from Nigeria studied 173 dyspeptic patients both endoscopically and histologically and found that concordance between endoscopic gastritis and histologic gastritis was $94.4 \%$ [14], while one local study found that endoscopic diagnosis of dyspepsia poorly correlated with histopathological findings [10] and this may be due to the small number of patients included in the later study.

In an earlier systematic review concerning patients from middle east regions including Egypt, the prevalence of $H$. pylori was high in patients with histological features of gastritis [8], results that were also reported from different areas around the world $[12,14-16]$ and re-enforced by the results of this study, among all patients with histological features of gastritis $(\mathrm{N}=146,96.7 \%)$, the prevalence of $H$. pylori (detected in the histology ) was $69.2 \%(\mathrm{~N}=101)$ and this was reported earlier from our locality by Hosam and Emara [10] who reported rates of $51.4 \%$ and also from other geographic areas and this emphasizes $H$. pylori as the most common and most important cause of gastritis [17-19].

The most striking finding of this study is detection of $H$. pylori bacilli in patients who initially tested negative for this organism by stool $H$. pylori antigen. And this raises many questions. The first is regarding the accuracy of $H$. pylori stool antigen as a reliable diagnostic test for $H$. pylori infection. In fact the accuracy of $H$. pylori stool antigen test is affected by many things. Its efficacy is lower when the stool is unformed or watery, because $H$. pylori-specific antigens in the stool samples are diluted. Temperature (needs lower temperatures) and the interval between stool sample collection and measurement also affect the results [20].

The second is the value of gastric mucosal biopsy in apparently non-inflamed or minimally inflamed gastric mucosa of dyspeptic patients in absence of gross features as erosions or ulcer. In fact many authors $[10,21]$ found high prevalence of gastric mucosal inflammation among dyspeptic patients with apparently normal upper endoscopy.

The problem of dealing with apparently normal gastric mucosa in dyspeptic patients had been resolved by AGA. They recommended that patients undergoing upper endoscopy for dyspepsia as the sole indication, should be exposed for routine biopsies of the normal-appearing gastric body and antrum for the detection of $H$. pylori infection if the $H$. pylori infection status is unknown whatever the immune status of the patient and they suggested following the 5-biopsy Sydney System with all specimens placed in the same jar [21].

In our study gastric mucosal erosions are significantly higher in group II than group I and this may be related to dietary habits. These patients were more consumers of spicy and fast foods that are known triggers of gastric hyperacidity.

Another important finding of this study is the inability to detect $H$. pylori bacilli in the stained slides from patients with positive stool $H$. pylori antigen. This may be due to several reasons. The first may be the uneven distribution of the infection [22], especially if patients were taking PPIs for long period [23]. The second may be non-sufficient four samples policy and the need for more biopsies covering a wide area of gastric mucosa $[22,24,25]$ and the AGA recommended five biopsy sites including two from the gastric antrum, two from the gastric body and one from the incisura when detec- tion of H. pylori is an intent [26]. The third once again is the validity of $H$. pylori stool antigen as a reliable test for diagnosis of $H$. pylori infection. In fact the accuracy of $H$. pylori stool antigen test is comparable to UBT when performed under certain precautions: the usage of monoclonal antibodies rather than polyclonal ones and enzyme immunoassay versus immunochromatography, stoppage of PPIs and antibiotics 2 weeks prior to the test [23]. For patients complaining of severe symptoms, antacids or histamine- 2 receptor antagonists, which do not interfere with testing, are allowed [27]. For patients unable to stop PPI therapy 2 weeks prior to stool antigen testing, positive test results can be considered as true positive whereas negative results may represent false negatives and should be confirmed with repeat testing 2 weeks after stopping PPI therapy [23]. Although all patients in this study stopped the use of PPIs and antibiotics 4 weeks in advance we cannot guarantee that all patients were compliant with our instructions, particularly some of them were of low socioeconomic and educational class.

Another possible cause for this discrepancy is the low sensitivity of Geimsa stain in detecting $H$. pylori organisms in the gastric mucosa biopsies.

In conclusion, all patients with dyspepsia in this study had endoscopic evidence of gastritis regardless of their $H$. pylori status. Most of these patients had histologic evidence of gastric mucosal inflammation. Consequently, it may be advisable to perform endoscopy for patients with dyspepsia and obtain gastric mucosal biopsies for histopathologic examination even in absence of alarm manifestations.

\section{Acknowledgments}

The authors would thank all colleagues who helped in conducting this study.

\section{Funding}

None.

\section{Conflict of Interest}

None.

\section{References}

1. Tack J, Talley NJ, Camilleri M, Holtmann G, Hu P, Malagelada JR, Stanghellini V. Functional gastroduodenal disorders. Gastroenterology. 2006;130(5):1466-1479.

2. Drossman DA, Dumitrascu DL. Rome III: New standard for functional gastrointestinal disorders. J Gastrointestin Liver Dis. 2006;15(3):237-241.

3. Hunt RH, Xiao SD, Megraud F, Leon-Barua R, Bazzoli F, van der Merwe S, Vaz Coelho LG, et al. Helicobacter pylori in developing countries. World Gastroenterology 
Organisation Global Guideline. J Gastrointestin Liver Dis. 2011;20(3):299-304.

4. Correa P, Piazuelo MB. Natural history of Helicobacter pylori infection. Dig Liver Dis. 2008;40(7):490-496.

5. Price AB, Misiewicz JJ. Sydney classification for gastritis. Lancet. 1991;337(8734):174.

6. Ford AC, Marwaha A, Sood R, Moayyedi P. Global prevalence of, and risk factors for, uninvestigated dyspepsia: a meta-analysis. Gut. 2015;64(7):1049-1057.

7. Talley NJ, Vakil NB, Moayyedi P. American gastroenterological association technical review on the evaluation of dyspepsia. Gastroenterology. 2005;129(5):1756-1780.

8. Khedmat H, Karbasi-Afshar R, Agah S, Taheri S. Helicobacter pylori Infection in the general population: A Middle Eastern perspective. Caspian J Intern Med. 2013;4(4):745-753.

9. Abd-Elsalam S, El Nawasany S, Elkhalawany W, Awny S, Mansour L, Ali LA, Soliman S. Increasing rates of treatment failures with the standard triple therapy for helicobacter pylori: a unique and alternative treatment option is urgent. Indian Journal of Medical Research and Pharmaceutical Sciences. 2016;3(4):13-16.

10. Dawod HM, Emara MW. Histopathological assessment of dyspepsia in the absence of endoscopic mucosal lesions. Euroasian J Hepato-Gastroenterol. 2016;6(2):97102.

11. Gado A, Ebeid B, Abdelmohsen A, Axon A. Endoscopic evaluation of patients with dyspepsia in a secondary referral hospital in Egypt. Alexandria Journal of Medicine. 2015;51:179-184z.

12. Ayana SM, Swai B, Maro VP, Kibiki GS. Upper gastrointestinal endoscopic findings and prevalence of Helicobacter pylori infection among adult patients with dyspepsia in northern Tanzania. Tanzan J Health Res. 2014;16(1):1622.

13. Ohkusa T, Fujiki K, Takashimizu I, Kumagai J, Tanizawa T, Eishi Y. Endoscopic and histological comparison of nonulcer dyspepsia with and without Helicobacter pylori infection evaluated by the modified Sydney system. Am J Gastroenterol. 2000;95(9):2195-2199.

14. Ajayi AO, Ajayi EA, Solomon OA, Duduyemi B, Omonisi EA, Taiwo OJ. Corelation between the endoscopic and histologic diagnosis of gastritis at the Ekiti State university teaching hospital, Ado Ekiti, Nigeria. International Journal of Internal Medicine. 2015;4(1):9-13.

15. Shrivastava UK, Gupta A, Gupta A, Bhatia A. Role of helicobacter pylori in functional dyspepsia. Indian J Surg.
2004; 66:341-346.

16. Shrestha S, Paudel P, Pradhan GB, Shrestha L, Bhattachan CL. Prevalence study of $\mathrm{H}$. pylori infection in dyspeptic patients coming to Nepal Medical College Teaching Hospital, Jorpati, Kathmandu. Nepal Med Coll J. 2012;14(3):229-233.

17. Patel MS, Shah AA, Patel HP, Joshi DS. Study of incidence of helicobacter organisms in cases of gastritis. Gujarat Medical Journal. 2013; 68(2): 91-96.

18. AlenezyAKA, Hassan TMM. Heliclobacter pylori associated chronic gastritis: Endoscopic and pathological findings, comparative study. Int J Genet Mol Biol. 2014;6(2):23-28.

19. Mao T, Wang Y, Yin F, Zhao Q, Yang L, Ding X, Tian $Z$. Association of endoscopic features of gastric mucosa with helicobacter pylori infection in Chinese patients. Gastroenterol Res Pract. 2016;2016:6539639.

20. Shimoyama T. Stool antigen tests for the management of Helicobacter pylori infection. World J Gastroenterol. 2013;19(45):8188-8191.

21. Nwokediuko SC, Okafor OC. Gastric mucosa in nonulcer dyspepsia: a histopathological study of Nigerian patients. I J Gastroenetrol. 2007;5(2).

22. Miftahussurur M, Yamaoka Y. Diagnostic methods of helicobacter pylori infection for epidemiological studies: critical importance of indirect test validation. Biomed Res Int. 2016;2016:4819423.

23. Dore MP, Pes GM, Bassotti G, Usai-Satta P. Dyspepsia: when and how to test for helicobacter pylori infection. Gastroenterol Res Pract. 2016;2016:8463614.

24. Asaka M, Kato M, Takahashi S, Fukuda Y, Sugiyama T, Ota H, Uemura N, et al. Guidelines for the management of Helicobacter pylori infection in Japan: 2009 revised edition. Helicobacter. 2010;15(1):1-20.

25. van IMC, Laheij RJ, de Boer WA, Jansen JB. The importance of corpus biopsies for the determination of Helicobacter pylori infection. Neth J Med. 2005;63(4):141-145.

26. Yang YX, Brill J, Krishnan P, Leontiadis G. American gastroenterological association institute guideline on the role of upper gastrointestinal biopsy to evaluate dyspepsia in the adult patient in the absence of visible mucosal lesions. Gastroenterology. 2015;149(4):1082-1087.

27. Gatta L, Vakil N, Ricci C, Osborn JF, Tampieri A, Perna F, Miglioli M, et al. Effect of proton pump inhibitors and antacid therapy on 13C urea breath tests and stool test for Helicobacter pylori infection. Am J Gastroenterol. 2004;99(5):823-829. 